KYUNGPOOK Math. J. 53(2013), 661-680

http://dx.doi.org/10.5666/KMJ.2013.53.4.680

\title{
On the Variational Approach for Analyzing the Stability of Solutions of Evolution Equations
}

\author{
Hamdy I. Abdel-Gawad* and M. S. Osman \\ Department of Mathematics, Faculty of Science, Cairo University, Egypt \\ e-mail: hamdyig@yahoo.com and mofatzi@yahoo.com
}

ABSTRACT. The eigenvalue problems arise in the analysis of stability of traveling waves or rest state solutions are currently dealt with, using the Evans function method. In the literature, it had been shown that, use of this method is not straightforward even in very simple examples. Here an extended "variational" method to solve the eigenvalue problem for the higher order differential equations is suggested. The extended method is matched to the well known variational iteration method. The criteria for validity of the eigenfunctions and eigenvalues obtained is presented. Attention is focused to find eigenvalue and eigenfunction solutions of the Kuramoto-Slivashinsky and $(\mathrm{K}[\mathrm{p}, \mathrm{q}])$ equation.

\section{Introduction}

Traveling waves TW emerge as solutions of nonlinear evolution equations EE in varieties of systems in physics, chemistry and biological sciences [1-8]. They have numerous types of geometrical structures as fronts, backs, wave trains or elliptic waves. They are "permanent" nonlinear wave solutions for a wide class of nonlinear evolution equations in physics and in reaction diffusion equations describing biological and chemical systems. The stability analysis of these waves is crucial in the sense that, relative to chemical systems, it specifies to what extent resultants of a chemical reactions are well routed. If TW are unstable then absolute or convective instability may be inspected. The stability analysis is carried out as follows: the evolution equation is transformed to a TW one's, and by linearizing the evolution equation near the TW solutions obtained, gives rise to a linear equation in the disturbances. By bearing in mind finiteness of the boundary conditions of solutions at $\pm \infty$, or of periodic boundary conditions the resultant eigenvalue problem is solved. There have been some advances over recent years in the development of stability

* Corresponding Author.

Received April 4, 2012; accepted August 30, 2012.

2010 Mathematics Subject Classification: 65F15, 58J45.

Key words and phrases: An extended variational method, Stability analysis-Traveling wave solutions Kuramoto-Sivashinsky equation.

This research was supported by a grant from Ferdowsi University of Mashhad No. MP91281 MIM. 
techniques for nonlinear waves such as fronts, pluses and wave trains. A motivating objective behind these stability methods is to inspect the concepts that can be adopted in applications. One of these concepts is that the stability of TW solution depends on their geometrical structure, if they are monotone or not. That is the stability of fronts, pulses or elliptic TW, generated in a system is indeed different. This yields a relationship between the geometrical (or topological) structure of the wave itself and its stability properties. By this analog, one can discriminate between stable and unstable waves in this system. Another example is the Vakhitov-Kolokov criterion [15, 16], which is applicable to systems such as the nonlinear Schrödinger equation. The existence of such rules for nonlinear waves with more complicated structure had motivated much of the research in this area.

For a set of (vector) EE the problem is too complicated. So that, solutions were obtained mainly, numerically in the literature by using the Evans function method [5]. It had been proved that this function is analytic and free from the independent variable in the equations for the spectral problems. It depends only on the eigenvalue parameter and the roots of this function produce the required solutions $[6,9]$. The formulation of this method requires writing the linearized equations as a system of first order ODE. The system obtained is then solved by using exponential dichotomy. It had been shown that the use of the Evans function is not straightforward [10].

The numerical computation of the Evans function is not straightforward as the boundary conditions are taken at finite values of the space variable. A drawback of this approximation is that pseudo-eigenvalues may emerge in numerical computations.

To overcome the lack of information about eigenvalues, further numerical algorithms had been presented [11]. They are based on the compound matrix where exterior algebra is involved .

Different approaches had been proposed which are based on various aspects; Approaches of topological (or geometrical structure) invariance of solutions (due to small disturbances when linearizing an EE) [12, 13, 14, 20].

Our aim here is to present an approach that fills the gap between the abstract approach and the applications. It is based mainly on the invariance of geometrical structure of TW solutions under small disturbances and the canonical transformations. A variational approach is then proposed and for numerical objectives, this approach is matched to the variational iteration method.

\section{General Formulation}

We consider an evolution equation as

$$
v_{t}+F_{0}\left(v, v_{x}, v_{x x}, v_{x x x}, \ldots, v_{x}^{(j)}\right)=0
$$


The equation (2.1) is translational invariant in space and time, the it admits a traveling wave solution. To this end, we make the transformations $t=t$ and $z=x-c t$, and (2.1) becomes

$$
v_{t}-c v_{z z}+F\left(v, v_{z}, v_{z z}, v_{z z z}, \ldots, v_{z}^{(j)}\right)=0 .
$$

By setting $v_{t}=0$, we get

$$
-c v_{o}^{\prime}+F\left(v_{o}, v_{o}^{\prime}, v_{o}^{\prime \prime}, v_{o}^{\prime \prime \prime}, \ldots, v_{o}^{(j)}\right)=0, v_{o}^{\prime}=\frac{d v_{o}}{d z} .
$$

The integrability of (2.1) and (2.3) are performed by using the Painleve' test in [18] and [19]. If the equation (2.3) is (completely or partially) integrable then explicit solutions $v_{o}$ could be found. We mention in some specific cases of nonintegrable equation (2.3), a particular solution may be obtained. There are a variety of methods for finding these solutions [17]. To study the stability of TW solutions $v_{o}=v_{o}(z)$, we linearize (2.2) near these solutions, namely

$$
v(z, t)=v_{0}(z)+V(z) e^{\lambda t},
$$

and by assuming that the function $F$ is continuously differentiable in the arguments, we get

$$
\lambda V-c V^{\prime}+A_{0}(z) V+A_{1}(z) V^{\prime}+A_{2}(z) V^{\prime \prime}+\ldots+A_{j}(z) V^{(j)}=0, A_{i}(z)=\frac{\partial^{i} F}{\partial v_{o}^{(i)}} .
$$

The eigenvalue problem (2.5) is subjected to the boundary conditions namely $V( \pm M)=0$. When $M \rightarrow \infty$, this holds for front, back or pulse waves but when $M$ is finite this corresponds to the case when the equation (2.3) assumes a periodic solution (or wave train). In the later case, those are periodic boundary conditions and they may be written $V( \pm M)=$ const. . We study the eigenvalue problem (2.5) by distinguishing two cases namely when (i) $j=2$ (for the second order case) and (ii) $j \geq 3$ (for higher orders). In each case we may consider $M \rightarrow \infty$ or $M$ is finite. Here, attention is focused to the case when $M \rightarrow \infty$.

Here the solution of the eigenvalue problem (2.5) when $j=2$ is treated by using the variational approach while when $j \geq 3$, an extended variational approach is used. These two approaches are matched to the variational iteration method (VIM).

We mention that $\lambda=0$ is an eigenvalue of (2.5) as when differentiating (2.3) with respect to $z$ and comparing the resultant equation with (2.5), we find that they are identical when (the eigenfunction) $V=v_{o}^{\prime}$. In fact this result is a direct 
consequence of the topological invariance of solutions. For $\lambda \neq 0$, eigenfunctions may be constructed, namely $V(z)=V^{m}(z)$ that correspond to $\lambda^{(m)}$ where $m \epsilon Z^{+}$, which is done by extrapolating the known eigenfunction $V=v_{o}^{\prime}$. The constructed eigenfunctions are taken as zero approximations in the iteration scheme of the VIM. In fact an appropriate selection of zero approximation are crucial for adjusting a good error tolerance in higher "order" approximate solutions [25].

Now we return to (2.5) and consider the two cases when $j=2$ and $j \geq 3$ mentioned in the above.

(i) When $j=2$, in this case we are concerned with a Sturm-Liouville boundary value problem where (2.5) may be rewritten in the self-adjoint form. By considering the cases of infinite or finite values of $M$, we have

$\left(i_{1}\right)$ When $M \rightarrow \infty$, the spectral set for TWS is determined by rewriting (2.5) as a variational equation, namely

$$
\lambda=\min _{V} \frac{\operatorname{Lim}_{M \rightarrow \infty} \int_{-M}^{M} P(z)\left(V^{\prime 2}-\frac{A_{0}(z)}{A_{2}(z)} V^{2}\right) d z}{\operatorname{Lim}_{M \rightarrow \infty} \int_{-M}^{M} \frac{P(z)}{A_{2}(z)} V^{2}(z) d z}, P(z)=e^{\int \frac{c z-A_{1}(z)}{A_{2}(z)} d z} .
$$

We point out that in (2.6) it was assumed that the two limits exist. When these two limits do not exist, then (2.6) is rewritten in the "weak" sense as

$$
\lambda=-\min _{V} \operatorname{Lim}_{M \rightarrow \infty} \frac{\int_{-M}^{M}\left(V\left(P(z) V^{\prime}\right)^{\prime}+\frac{A_{0}(z)}{A_{2}(z)} V^{2}\right) d z}{\int_{-M}^{M} \frac{P(z)}{A_{2}(z)} V^{2}(z) d z} .
$$

The variational method asserts that a minimizing sequence $\left\{V_{n}\right\}$ exists and for a classes of functions $\left\{V_{1}\right\} \subset\left\{V_{2}\right\} \subset \ldots$, and it holds that $\lambda^{(1)} \geq \lambda^{(2)} \geq \ldots[21]$.

$\left(i_{2}\right)$ When $\mathrm{M}$ is finite the equation (2.6) holds formally by removing the limits.

It is worth noticing that although (2.6) and (2.7) are variational equations but they also hold also exactly. Direct consequences from these equations are as follows. We remark that the equations (2.6) and (2.7) are in general transcendental in $\lambda$, so that solutions may be be real, complex or pure imaginary. Indeed the equation (2.6) solves the eigenvalue problem for the stability of pulses ( when $M \rightarrow \infty$ ) or for periodic solutions ( when $M=$ consts.). While (2.7) solves the eigenvalue problem for front or back waves. We remark that in this later case (2.7) is not transcendental in $\lambda$, which is a direct result from the limit rules, so that the eigenvalues are all real. This does not hold in the former case.

It is worth noticing that (2.6) or $((2.7))$ are constructed from (2.5) by recasting higher order derivative terms into one term. By bearing this idea in mind, we have

(ii) When $j \geq 3$, We extend the use of the variational approach for the solution of the eigenvalue problem (2.5). In this case, terms of second and first order derivatives are also grouped together and recasted to one term. 
$\left(i i_{1}\right)$ In case of absence of second order term and when $M \rightarrow \infty$, by a direct integration, after multiplying by $V$, and by using that mentioned in the above, then we get respectively, according to if the two limits exists or they do not exist,

$$
\begin{gathered}
\lambda=\frac{\operatorname{Lim}_{M \rightarrow \infty} \int_{-M}{ }^{M} P(z)\left(V^{\prime 2}-\ldots\right) d z}{\operatorname{Lim}_{M \rightarrow \infty} \int_{-M}{ }^{M} \frac{P(z)}{A_{2}(z)} V^{2}(z) d z}, P(z)=e^{\int \frac{c z-A_{1}(z)}{A_{2}(z)} d z} \\
\quad \lambda=-\operatorname{Lim}_{M \rightarrow \infty} \frac{\int_{-M}{ }^{M}\left(V\left(P(z) V^{\prime}\right)^{\prime}+\ldots\right) d z}{\int_{-M}{ }^{M} \frac{P(z)}{A_{2}(z)} V^{2}(z) d z} .
\end{gathered}
$$

We mention that the terms that are expressed by the dots in (2.8) are too lengthy and they could be obtained directly by using (2.5). So that it is irrelevant that they are written here.

$\left(i i_{2}\right)$ When $M$ is finite, the integrals in the RHS of (2.8) exist, so that the first equation (2.8) holds, by removing the limits.

We return to the construction of eigenfunctions (as zero approximations), and bear in mind that $V(z)=v_{0}^{\prime}(z)$ is an eigenfunction that corresponds to $\lambda=0$. It is rewritten (or expanded) in terms of orthogonal polynomials that fit with boundary conditions, namely $V(z)=v_{0}^{\prime}(z)=\sum_{k>m_{0}} a_{k} P_{k}^{m_{0}}(\xi)$, where $P_{k}^{m_{0}}(\xi)$ is the associated Legendre polynomial where $\xi:=\xi(z)$. This fits with the different geometrical structure of TWS of (2.3); front (or many fronts), backs or pulses, with $\xi=\tanh (. z)$. When the solution of (2.3), namely $v_{0}(z)$ is periodic, then $V(z)=v_{0}^{\prime}(z)$, is expanded also in $P_{k}^{m_{0}}(\xi)$ but here $\xi=\cos (. z)$ (or $\xi=\sin (. z)$ ) to fit with periodic conditions. It is worth noticing that in this case $m_{0}$ is fixed.

When $\lambda \neq 0$ we let $m_{0}$ varies and suggest that $V(z)=V^{(m)}(z)$. By substituting into (2.6), we get $\lambda^{(m)}$. Indeed the pair $\left(\lambda^{(m)}, V^{(m)}(z)\right)$ do not satisfy exactly the equation (2.5) and is considered as the zero order approximation to the solution of the eigenvalue problem. For higher order approximations we use an appropriate numerical method which is taken here the VIM. Thus approach for evaluating the eigenvalues presented in above, namely the equations (2.6)-(2.8), is matched to the variational iteration method VIM [25]. To this end, we write the approximate solution to the required pair as $\left(V_{n}^{(m)}(z), \lambda_{n}^{(m)}\right)$ together with the zero approximation, $\left(V_{0}^{(m)}(z), \lambda_{0}^{(m)}\right)$ that was constructed in the above. The VIM suggests to write a discretized form for $(2.5)$ and on a finite interval $[-M, M]$, we have

$$
\begin{gathered}
V_{n}^{(m)}=V_{n-1}^{(m)}+\int_{-M}^{z} \eta\left(z_{1}\right) R_{n-1}^{(m)} d z_{1}, R_{n-1}^{(m)} \equiv R_{n-1}^{(m)}\left(z, V_{n-1}^{(m)}, \ldots, V_{n-1}^{(m)(j)}\right), \\
R_{n-1}^{(m)}=L_{0}\left(V_{n-1}^{(m)}, V_{n-1}^{(m) \prime}, . .\right)+L_{1}\left(z, \widetilde{V}_{n-1}^{(m)}, \widetilde{V}_{n-1}^{(m) \prime}, . .\right), n \geq 1
\end{gathered} .
$$

In (2.9) $R_{n}^{(m)}:= \pm\left|E_{n}^{(m)}\right|$ stands for the left hand side of (2.5) evaluated at $V_{n}^{(m)}$, and $\left|E_{n}^{(m)}\right|$ is the local error (or defect error [26]). $L_{0}(\ldots)$ is in general linear in the arguments and $\delta \widetilde{V}_{n}^{(m,)}=0$ is " the restricted variation". The function $\eta(z)$, the 
Lagrange multiplier, is determined by the differential equation that results from the variational equation $\delta V_{n}^{(m)}=0$ and the boundary conditions. We mention that (2.9) may hold on an infinite interval by taking the limit $M \rightarrow \infty$ if the exists.

By the matching process, a discretization in the equation that determines the eigenvalues, namely in the equations (2.6)-(2.8), is done. For instance, we consider the equation (2.8) which becomes

$$
\begin{gathered}
\lambda_{n}^{(m)}=\frac{\operatorname{Lim}_{H \rightarrow \infty} \int_{-H}^{H} P_{n}^{(m)}(z)\left(V_{n}^{(m) \prime 2}-\ldots\right) d z}{\operatorname{Lim}_{H \rightarrow \infty} \int_{-H}^{H} \frac{P_{n}^{(m)}(z)}{A_{2 n}^{(m)}(z)} V(m)_{n}^{2}(z) d z}, P_{n}^{(m)}(z)=e^{\int \frac{c z-A_{1 n}^{(m)}(z)}{A(m)_{2 n}(z)} d z} \\
\lambda_{n}^{(m)}=-\operatorname{Lim}_{H \rightarrow \infty} \frac{\int_{-H}^{H}\left(V_{n}^{(m)}\left(P_{n}^{(m)}(z) V_{n}^{(m) \prime}\right)^{\prime}+\ldots\right) d z}{\int_{-H}{ }^{H} \frac{P_{n}^{(m)}(z)}{A_{2 n}^{(m)}(z)} V_{n}^{(m) 2}(z) d z} .
\end{gathered}
$$

It is worth noticing that the construction of (or families of) eigenfunctions, as zero approximations, is not unique. So that we need a criteria to test for validity if the constructed set of eigenfunctions and the corresponding set of eigenvalues, namely $\left(\left\{V_{0}^{(m)}\right\},\left\{\lambda_{0}^{(m)}\right\}\right), m \epsilon \mathbb{Z}^{+}$are the right zero-approximate ones . We suggest that the pair $\left(\left\{V_{0}^{(m)}\right\},\left\{\lambda_{0}^{(m)}\right\}\right), m \in \mathbb{Z}^{+}$is the right zero-approximation if the set $\left(\left\{V_{n}^{(m)}\right\},\left\{\lambda_{n}^{(m)}\right\}\right), m \in \mathbb{Z}^{+}$converges, namely to $\left(\left\{V_{\infty}^{(m)}\right\},\left\{\lambda_{\infty}^{(m)}\right\}\right), m \in \mathbb{Z}^{+}$. Thus the criteria is based on the convergence of the iterated set of solutions. This one of the main results in this work.

To this end the convergence theorem for the VIM is invoked and its proof is given in what follows.

For this aim and also for analyzing the error bound in the VIM, we assume for simplicity that $L_{0}(V, \ldots)=-c \frac{\partial V}{\partial z}$ and $L_{1}(V, \ldots)$ is given by the rest terms in the RHS of (2.5). By bearing this in mind and in (2.9) we $\operatorname{set} \delta V_{n}^{(m)}=0$ to get an equation in $\eta(z)$ that solves $\operatorname{to} \eta(z)=1 / c$. For details see sections 4 and 5 . We assume that $\mathrm{S}$ is the set of approximate solutions of (2.9), for a fixed $m$, with tolerable error ; $S=\left\{V_{n}^{(m)},\left\|V_{n}^{(m)}-V^{(m)}\right\|<\varepsilon\right\}$. It is worthy noticing that $\left\|V_{n}^{(m)}-V^{(m)}\right\|=\left\|e_{n}^{(m)}\right\|$ is the :direct" step-wise error, and $\operatorname{Max}_{n}\left\|V_{n}^{(m)}-V^{(m)}\right\|$ is the absolute error.

We mention that $\mathrm{S}$ is closed under addition and multiplication by $\alpha,|\alpha|<1$. $S$ is endowed by the norm $\|\cdot\|_{S}=\operatorname{Sup}_{V_{n}^{(m)} \epsilon S}\left\|V_{n}^{(m)}\right\|,\left\|V_{n}^{(m)}\right\|=\operatorname{Sup}_{|z|<M} \mid$ $V_{n}^{(m)}(z) \mid$ and $\left\|E_{n}^{(m)}\right\|=\operatorname{Sup}_{z}\left|E_{n}^{(m)}(z)\right|$, where $\left|E_{n}^{(m)}(z)\right|= \pm R_{n}^{(m)}\left(V, V_{z}, ..\right)$, is the "indirect" step-wise error, and $R_{n}^{(m)}\left(V, V_{z}, ..\right)$ was defined in the above. By bearing (2.9) in mind we define the mapping $M$ :on $S ; M\left(V_{n}^{(m)}\right)=V_{n+1}^{(m)}$ where $V_{n+1}^{(m)}$ is given by the RHS of (2.9). Hereafter, for convenience the variable $z$ will be omitted. We show that under appropriate conditions $M: S \rightarrow S$. 
Lemma 2.1. If $R_{n}^{(m)}\left(z, V_{n}^{(m)},\left(V_{n}^{(m)}\right)^{\prime}, ..\right)$ is global Lipschitz condition with constant $\mu$ and $M<1 / 2 \mu$, then $\mathrm{M}$ maps $S$ into $S$.

Proof. It suffices to show that $V_{n+1}^{(m)} \epsilon S$ and the rest of the proof is is done as in convergence theorem.

The following lemma holds

Lemma 2.2. If $V_{0}^{(m)} \epsilon C^{k}([-H, H])$, Then (i) $V_{n}^{(m)} \epsilon C^{k}([-H, H]), n=1,2, \ldots$ (ii) $V_{n}^{(m)}(-H)=V_{0}^{(m)}(-H)$. (iii) $M($.$) is monotone.$

Proof. (i) and (ii) hold directly by induction .

(iii) We assume that $V_{n}^{(m)(1)}, V_{n}^{(m)(2)} \epsilon S, V_{0}^{(m)(1)}=V_{0}^{(m)(2)}=V_{0}^{(m)}$ then $M\left(V_{1}^{(m)(1)}\right)=M\left(V_{1}^{(m)(2)}\right)$ But when $n=1$ and in (2.9), it holds that $M\left(V_{1}^{(m)(1)}\right)=$ $M\left(V_{1}^{(m)(2)}\right)$ iff $V_{1}^{(m)(1)}=V_{1}^{(m)(2)}$. At $n=k$, we assume that $M\left(V_{k}^{(m)(1)}\right)=$ $M\left(V_{k}^{(m)(2)}\right)$ iff $V_{k}^{(m)(1)}=V_{k}^{(m)(2)}$. Also from (2.9) it holds that $M\left(V_{k+1}^{(m)(1)}\right)=$ $M\left(V_{k+1}^{(m)(2)}\right)$ iff $V_{k+1}^{(m)(1)}=V_{k+1}^{(m)(2)}$. Thus $M($.$) is monotone.$

In what follows we give a "new "proof to the theorem of convergence to the VIM and also analyze the error bounds for solutions that depend basically on the error associated with the zero approximation. For instance the dicretization of (2.5) is written in the form

$$
R_{n}^{(m)}=-c V_{n}^{(m) \prime}+\left(\lambda V_{n}^{(m)}+A_{n 0}^{(m)} V_{n}^{(m)}+A_{n 1}^{(m)} V_{n}^{(m) \prime}+\ldots+A_{n j}^{(m)} V_{n}^{(m)(j)}\right)
$$

where $A_{n i}^{(m)}=\frac{\partial R_{n}^{(m)}}{\partial V_{n}^{(m)(i)}}$. We proceed to the proof and state that a function $g($. is globally Lipschitz continuous if $g($.$) and g \prime($.$) are locally Lipschitz continuous.$ Here this definition is extended and we assume that $g^{(i)}(),. i=0,1, \ldots, j$ are locally Lipschitz conditions.

\section{Convergence Theorem and the Error Bound}

In what follows, we give the convergence theorem for VIM.

Theorem 3.1. If $A_{i n}^{(m)}(\ldots), i=0,1, \ldots, j$, are locally Lipschitz continuous for all $\mathrm{n}$, and $V_{0}^{(m)} \epsilon C^{k}([-\varepsilon / K, \varepsilon / K])$, where $K$ is the Lipschitz constant and $0<\varepsilon<1$, then the sequence of solutions $V_{n}^{(m)} n \geq 1$ converges uniquely to the exact solution $V^{(m)}$. 
Proof. From the first assumption, it holds that the function $R_{n}^{(m)}$ is global Lipschitz condition so that

$$
\left|R_{n}^{(m)}\left(z, V_{n}^{(m)}, \ldots, V_{n j}^{(m)}\right)-R_{k}^{(m)}\left(z, V_{k j}^{(m)}, \ldots, V_{k j}^{(m)}\right)\right| \leq \mu\left|V_{n}^{(m)}-V_{k}^{(m)}\right|
$$

As $\eta(z)=-1$, and from (ii) of lemma 2.2, the equation (2.9), for $n \geq 0$, is rewritten as

$$
V_{n+1}^{(m)}(z)=V_{0}^{(m)}(-H)+\int_{-M}^{z} R_{n}^{(m)}\left(z, V_{n}^{(m)}, \ldots, V_{n j}^{(m)}\right) d z_{1} .
$$

From (3.2) we have

$$
\begin{gathered}
\left|M\left(V_{n}^{(m)}\right)-M\left(V_{k}^{(m)}\right)\right| \leq \int_{-M}^{z} \mid R_{n}^{(m)}\left(z_{1}, V_{n}^{(m)}\left(z_{1}\right), \ldots, V_{n j}^{(m)}\left(z_{1}\right)\right) \\
-R_{k}^{(m)}\left(z_{1}, V_{k}^{(m)}\left(z_{1}\right), \ldots, V_{k}^{(m)(j)}\left(z_{1}\right)\right) \mid d z_{1} \leq \\
\int_{-M}^{M}\left|R_{n}^{(m)}\left(z_{1}, V_{n}^{(m)}\left(z_{1}\right), \ldots, V_{n j}^{(m)(j)}\left(z_{1}\right)\right)-R_{n}^{(m)}\left(z_{1}, V_{k}^{(m)}\left(z_{1}\right), \ldots, V_{k j}^{(m)}\left(z_{1}\right)\right)\right| d z_{1} .
\end{gathered}
$$

By using the equation (3.1), it holds that

$$
\left\|M\left(V_{n}^{(m)}\right)-M\left(V_{k}^{(m)}\right)\right\| \leq 2 \mu M\left\|V_{n}^{(m)}-V_{k}^{(m)}\right\| .
$$

In the equation (3.4), when $M=\varepsilon / 2 \mu, 0<\varepsilon<1$, we get

$$
\left\|M\left(V_{n}^{(m)}\right)-M\left(V_{k}^{(m)}\right)\right\| \leq \varepsilon\left\|V_{n}^{(m)}-V_{k}^{(m)}\right\|, 0<\varepsilon<1 .
$$

Thus the mapping $M$ is a contraction mapping and also it is monotone. By the fixed point theorem, then the sequence of solutions given by $(2.9)$ has a unique fixed point,. Thus $\operatorname{Lim}_{n \rightarrow \infty} V_{n}^{(m)}=V^{(m)}$. This proof is completed.

Corollary. The sequence of solutions $V_{n}^{(m)}(z)$ converges uniformly to $V^{(m)}(z)$ on $\left[-H_{1}, H_{1}\right], H_{1}<M<\varepsilon / 2 \mu$.

Proof. From the second condition in theorem 3.1, it holds that $\operatorname{Lim}_{n \rightarrow \infty} V_{n}^{(m)(i)}=$ $V^{(m)(i)}, i=1, \ldots, j$ Also as $A_{i n}^{(m)}(\ldots)$ is uniformally bounded then it holds that $\operatorname{Lim}_{n \rightarrow \infty} A_{n i}^{(m)}=A_{i}^{(m)}$, Consequently $\operatorname{Lim}_{n \rightarrow \infty}\left|R_{n}^{(m)}\left(V_{n}^{(m)}(z), \ldots\right)\right|=0,-M \leq$ $z \leq M$. Thus by taking the limit in the equation (2.9) we have $\operatorname{Lim}_{n \rightarrow \infty} V_{n}^{(m)}(z)=$ $V^{(m)}(z)$ uniformly on $-M \leq z \leq M$. 
An alternative proof for the point wise-convergence theorem for the VIM had been given in [24].

Theorem 3.2. The sequence $\left\{\lambda_{n}^{(m)}\right\}$ converges uniformly to the exact solution $\lambda^{(m)}$ of $(2.8)$.

The proof is direct as from theorem 3.1, it holds that the limits when $n \rightarrow \infty$ of the denominator and of the numerator in the RHS of (2.10) exist.

For the stability criteria, we have the following lemma.

Lemma. If $\lambda_{n} \rightarrow \lambda$ when $n \rightarrow \infty$, then the TWS is unstable if $\lambda>\lambda_{0}>0$ and it is stable if $\lambda<\lambda_{0}<0$, where $\lambda_{0}$ is the eigenvalue that corresponds to the zero approximation.

\section{1.the error bound}

To analyze the error bound for the VIM, we start by finding the rate of convergence.

1- Rate of convergence.

From (3.5) we have

$$
\begin{gathered}
\left|V_{n+1}^{(m)}(z)-V_{k+1}^{(m)}(z)\right|<\varepsilon\left|V_{i, n}^{(m)}(z)-V_{i, k}^{(m)}(z)\right|, \\
-M<z<\frac{\varepsilon}{2 \mu}, 0<\varepsilon<1, i=1, \ldots, m
\end{gathered}
$$

and from the convergence theorem, it holds that

$$
\left\|V_{n+1}^{(m)}-V^{(m)}\right\| /\left\|V_{n}^{(m)}-V^{(m)}\right\| \rightarrow \varepsilon_{0} \text {, as } n \rightarrow \infty,
$$

and $0 \leq \varepsilon_{0} \leq \varepsilon<1$. Thus the convergence could not be super linear and it is sub-linear when $\varepsilon_{0}=0$.

2- Error bound for two successive solutions

From the equation (3.2) and by using the q-mean value theorem, it holds that

$$
\left|E_{n}^{(m)}(z)-E_{n-1}^{(m)}(z)\right|<(z+M)\left|E_{n-1}^{(m)}(\xi)\right|,-M<\xi<z .
$$

3 - Error bounds is related to the error in the zero approximation.

The equation (2.9) can be rewritten in the form

$$
V_{n}^{(m)}(z)-V_{0}^{(m)}(t)=\mp \sum_{j=0}^{j=n-1} \int_{-M}^{z}\left|E_{, j}^{(m)}(s)\right| d z_{1} .
$$


It holds that

$$
\begin{gathered}
(n-1)(z+M)\left\|E_{0}^{(m)}(\xi)\right\|<\left\|E_{n}^{(m)}(z)-E_{0}^{(m)}(z)\right\|<n(z+M)\left\|E_{0}^{(m)}(\xi)\right\|, \\
-H<\xi<z, i=1, \ldots, m .
\end{gathered}
$$

To summarize, an algorithmic form for the method is presented as follows:

(i) Given the eigenfunction $V^{\left(m_{0}\right)} \equiv V^{\left(m_{0}\right)}(\xi) \equiv v_{0}^{\prime}(\xi), \xi:=\xi(z)$ that corresponds to the eigenvalue $\lambda=0$. It is expanded in terms of the associated Legendre polynomials, namely $V^{\left(m_{0}\right)}(\xi)=v_{0}^{\prime}(\xi)=\sum_{j=k_{0}}^{j=n_{0}} a_{j} P_{j}^{k_{0}}(\xi)$, for some $k_{0}$ and $n_{0}>k_{0}$.

(ii) For $\lambda \neq 0$, construct the set of zero-eigenfunctions approximations ( namely, $\left.V_{0}^{(m)}(\xi)\right)$, so that when $m=m_{0}$, it reduces to $V^{\left(m_{0}\right)}(\xi)$ in the step (i) and fix $m$.

(iii) Use (2.10) to compute the eigenvalue, namely $\lambda_{0}^{(m)}$.

(iv) Use (2.9) to compute $V_{1}^{(m)}$ and use (2.10) to compute $\lambda_{1}^{(m)}$.

(v) When $\left\|E_{1}^{(m)}\right\| /\left\|V_{1}^{(m)}\right\|<\left\|E_{0}^{(m)}\right\| /\left\|V_{0}^{(m)}\right\|$ and $\left|\lambda_{1}^{(m)}-\lambda_{0}^{(m)}\right|<\left|E_{1}\right|$, where $\left|E_{1}\right|$ is a tolerable error, set $n=2$ and continue.

(vi) Return to step (ii) and change the value of $m$.

It worth noticing that the conditions in the step (v) are necessary (but they are not sufficient) for the pair $\left(V_{n}^{(m)}, \lambda_{n}^{(m)}\right)$ to converge when $n \rightarrow \infty$.

\section{Applications}

\section{1. the Kuramoto-Sivashinsky equation (KS)}

The KS equation arises in different areas in physics $[14,15,16]$ and it reads

$$
u_{t}+\gamma u_{x x}+u_{x x x x}+u u_{x}=0,0<\gamma \leq 1 .
$$

The TW solution results from the equation

$$
u_{t}-c u_{z}+\gamma u_{z z}+u_{z z z z}+u u_{z}=0,
$$

by setting $u_{t}=0$ in (4.2). The reduced equation solves to $[15,16]$

$$
u_{0}(z)=c-\frac{15}{19} \sqrt{\frac{11}{19}} \gamma^{\frac{3}{2}} \tanh (b z)\left(9-11 \tanh ^{2}(b z)\right), z=x-c t
$$

where $k=\sqrt{\frac{11 \gamma}{76}}$. The equation (4.3) is a solitary wave solution and it shows a pulse coupled to a wave front.

By using (4.2), the equation that determines the eigenvalues is given by 


$$
R(U):=U^{\prime \prime \prime \prime}+\gamma U^{\prime \prime}+\left(u_{0} U\right)^{\prime}-c U^{\prime}+\lambda U=0,
$$

where the boundary conditions are $U( \pm \infty)=0$ and $U^{\prime}( \pm \infty)=0$. By using (4.5), we can verify that when $U=u_{0}^{\prime}$, we find that $\lambda=0$.

In the equation (4.4), we remark that $u_{0}-c$ is free from $c$ so that $\lambda$ does not depend explicitly on $c$.

In (4.4) as each term is a exact derivative, the last two terms are recasted into on term so that it may be rewritten in the form

$$
-c\left(e^{-\frac{\lambda z}{c}} U\right)^{\prime}+\left(U^{\prime \prime \prime}+\gamma U^{\prime}+u_{0} U\right)^{\prime} e^{-\frac{\lambda z}{c}}=0 .
$$

In (4.5) by taking into our consideration that zero-eigenfunction solutions could be constructed by using (4.3). It is worth noticing the validity of this choice will be verified in the sense mentioned in section2 (after the equation (2.10)). By substituting into the second equation (2.8), we get an implicit equation for the eigenvalue $\lambda$, namely

$$
\lambda=-2 \operatorname{Lim}_{R \rightarrow \infty} \frac{\int_{-R}^{R}\left(U^{\prime \prime \prime}+\gamma U^{\prime}+u_{0} U\right)^{\prime} U e^{-\frac{\lambda z}{c}} d z}{\int_{-R}^{R}\left(U^{2} e^{-\frac{\lambda z}{c}}\right) d z} .
$$

The equation (4.6) is a transcendental equation which undertakes the fact that the limits in the numerator and the denominator do not exist. By bearing this in mind we have the following lemma.

Lemma 4.1. The equation (4.6) (or (4.4)) has real eigenvalue solutions. This holds by using the limits rules.

To construct eigenfunction for (3.4) we start by the particular solution pair $\left(U=u_{0}^{\prime}, \lambda=0\right)$ to (3.4) and generalize the known eigenfunction. To this end we use the step (i) in the algorithm, that is we write the function $u_{0}^{\prime}$ as a series in the associated Legendre Polynomial, namely

$$
U:=u_{0}^{\prime}=-\frac{33}{2527} \gamma^{2}\left(25 P_{2}^{2}(\xi)-11 P_{4}^{2}(\xi)\right)
$$

After the step (i) in the algorithm presented in section 2, we find that $k=2$.

Thus we have

$$
U_{0}^{(m)}=-\frac{33}{2527} \gamma^{2}\left(25 P_{2}^{m}(\xi)-11 P_{4}^{m}(\xi)\right), m=0,1,2 .
$$

We mention that in the case $m=0$, the eigenfunction in (4.8) does not satisfy the boundary conditions in (4.4). Also when $m=2$, we get $\lambda=0$ and $U_{0}^{(2)}=U$. 
Thus, we consider the case when $m=1$ into (4.8) and get

$$
U_{0}^{(1)}=-\frac{33}{2527} \gamma^{2}\left(25 P_{2}^{1}(\xi)-11 P_{4}^{1}(\xi)\right)
$$

To evaluate the eigenvalue that corresponds to eigenfunction $U_{0}^{(1)}$ which is given in (4.9), we use the step (iii) to calculate $\lambda_{0}^{(1)}$. To this end we use (4.6). It worth noticing that the integrals in (4.6) do not exist, so that it is used in the "weak" sense as in (2.7) and get a direct result for the eigenvalue as

$$
\lambda_{0}^{(1)}=\frac{98395}{2085136} \gamma^{2}
$$

From (4.10) we find that $\lambda_{0}^{(1)}>0$. By using (4.10) and (4.9) into (4.4), by substituting $U_{0}^{(1)}$ for $U$ and $\lambda_{0}^{(1)}$ for $\lambda$. The norm of the relative error in the eigenfunction, is defined by $\left\|(R E)^{(1)}\right\|=\operatorname{Max}_{n}\left\|(R E)_{n}^{(1)}\right\|,\left\|(R E)_{n}^{(1)}\right\|=$ $\left\|U_{n+1}^{(1)}-U_{n}^{(1)}\right\|_{2} /\left\|U_{n}^{(1)}\right\|_{2}$ and a similar definition holds for $\lambda$. In this case, the recursion equation in the VIM is

$$
\begin{gathered}
\left.U_{n+1}^{(1)}=U_{n}^{(1)}+\int_{-\infty}^{z} \eta(y)\left(\widetilde{\left(\left(U_{n}^{(1)}\right.\right.}\right)^{\prime \prime \prime \prime \prime}+\gamma\left(U_{n}^{(1)}\right)^{\prime \prime}+\left(u_{0}-c\right) \widetilde{\left({U_{n}^{(1)}}^{(1)}\right.}\right)^{\prime} \\
\left.+\lambda_{n}^{(1)} U_{n}^{(1)}+\widetilde{u_{0}^{\prime}} \widetilde{U_{n}^{(1)}}\right) d y, n \geq 0,
\end{gathered}
$$

where $\eta(y)$ is the Lagrange multiplier. For simplicity the super script: (1) is dropped every where.

In (4.11), $\widetilde{U_{n}^{(1)}}$ is taken with a restricted variation; $\delta \widetilde{U_{n}}=0 . \quad$ By a direct calculation, we find that $\eta(y)$ satisfies the following differential equation

$$
\gamma \frac{d^{2} \eta}{d y^{2}}+\lambda \eta=0, \eta^{\prime}(z)=\frac{1}{\gamma}, \eta(z)=0
$$

To calculate $\lambda_{n}^{(1)}, n=1,2, \ldots$,we use the step (iv) in the algorithm.

When $n=1$, we evaluate $U_{1}^{(1)}$ by using (2.8) and (4.11). in this case we have

By calculating $U_{1}^{(1)}$ from (4.11) by using (4.9) and (4.12), it used to calculate $\lambda_{1}^{(1)}$ (cf. (4.6)) . The results for $\lambda_{i}^{(1)}$ and $\left\|(R E)_{i}^{(1)}\right\|, i=1,2$ are displayed against $\gamma$ and they are shown in figures 1 and 2 respectively. 

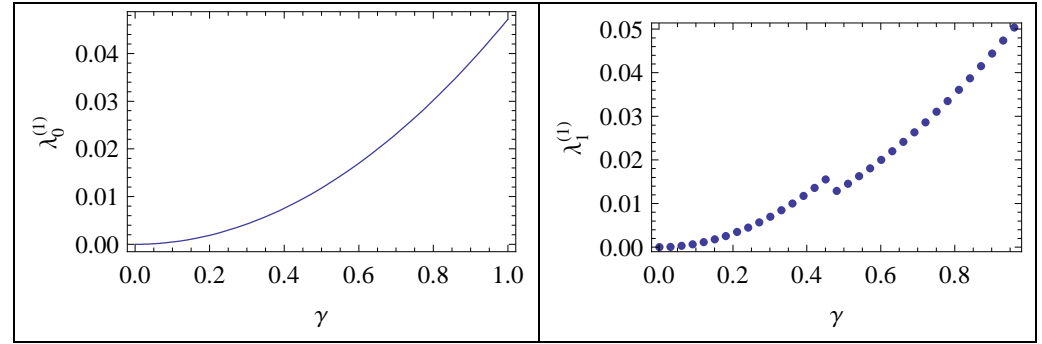

Figure 1
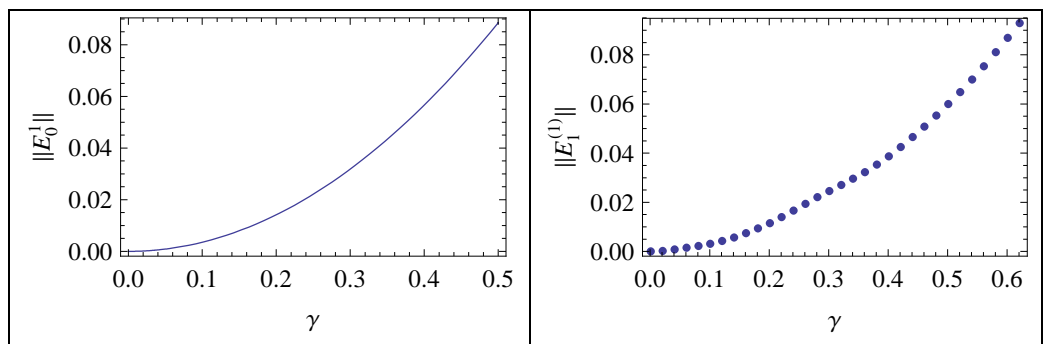

Figure 2

After figure 2, we find that $\left\|(R E)_{1}^{(1)}\right\|<\left\|(R E)_{0}^{(1)}\right\|$ and they are a tolerable relative errors.

By the remark made in section 2, the associated Legendre expansion in (4.7) is not unique. A second expansion exists and is given by

$$
U^{\left(k_{0}\right)}:=u_{0}^{\prime}=-\frac{11}{5054} \gamma^{2}\left(5 P_{4}^{4}(\xi)-56 P_{4}^{2}(\xi)\right)
$$

In this case $k_{0}=4$. Thus we have

$$
U^{(m)}=-\frac{11}{5054} \gamma^{2}\left(5 P_{m}^{4}(\xi)-56 P_{m}^{2}(\xi)\right), m \geq 4 .
$$

When $m=5$, we find that

$$
\lambda_{0}^{(5)}=\frac{60808}{130321} \gamma^{2},
$$

and also $\lambda_{0}^{(5)}>0$. We think in this case there exists an infinite number of eigenvalues. They satisfy the inequality $\lambda_{0}^{(5)}<\lambda_{0}^{(6)}<\ldots$. On the other hand we find that $\lambda=0$ is a simple eigenvalue. 
As $\lambda_{n}^{(m)}, n=0,1, \ldots$ are all positive and from theorem 2.2 it holds that $\lambda_{n}^{(m)}$ converges to $\lambda^{(m)}>0$, then the TW solution that given by (3.3) is unstable. So that, we investigate the behavior of the instability if it absolute, convective, diffusive or ultra-diffusive ones. To this end, the linearized solution of (4.1), which is given by

$$
u(z, t)=c_{o} u_{0}^{\prime}+c_{1} e^{\lambda^{(1)} t} U^{(1)}(z)+\sum_{m=5}^{\infty} c_{m} e^{\lambda^{(m)} t} U^{(m)}(z),
$$

is displayed in the space $(z, t, u)$. Merely the first few terms are taken at the zero approximation and the results are shown in figure 3 when $\gamma=0.2$ and $\gamma=0.5$ and $c_{i}=1$ for all $i$.

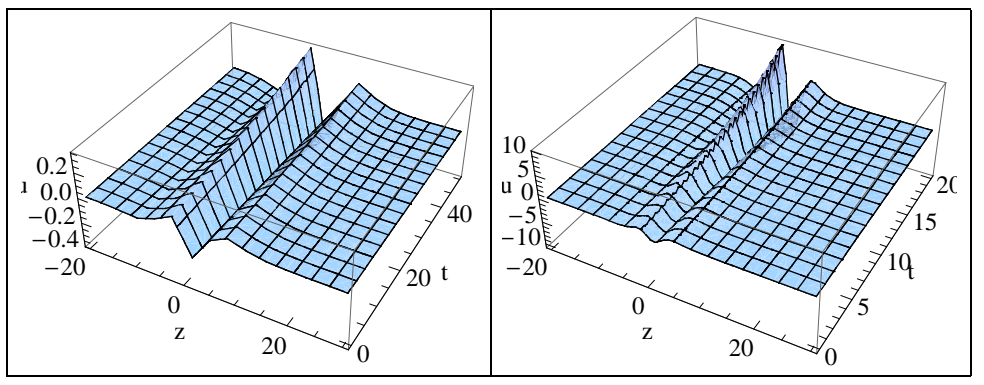

Figure 3. Shows the behavior of the linearized solution of the equation (4.2) when $\gamma=0.2$ (left) and $\gamma=0.5$ (right).

After figure 3, it holds that the solution undergoes an absolute instability, while diffusive effects are manifested in narrowing or enlarging the pulse (that coupled to a wave front).

\section{Stability of TWS of the Dispersive $K(p, q)$ Equation}

The dispersive $K(p, q)$ equation had been introduced in [22] to generalize the well-known $\mathrm{KdV}$ equation. It reads

$$
u_{t}+a\left(u^{p}\right)_{x}+\left(u^{q}\right)_{x x x}=0, p, q>1 .
$$

In a frame traveling with speed $c$, this equation becomes:

$$
u_{t}-c u_{z}+a\left(u^{p}\right)_{z}+\left(u^{q}\right)_{z z z}=0
$$

To get the TWS, we set $u_{t}=0$. The obtained equation has a first integral which is given by 


$$
-c u_{t}+a u^{p}+\left(u^{q}\right)^{\prime \prime}=C_{1}, u^{\prime}=\frac{d u}{d z},
$$

and $C_{1}$ is a constant. By taking $q=p$ and by a straightforward calculations, we get the solution (which was first given in [23]) as

$$
u_{0}(z)=\left\{\begin{array}{cl}
\left(\frac{2 c p}{a(p+1)}\right)^{\frac{1}{p-1}}\left(\cos \left(z / z_{0}\right)\right)^{\frac{2}{p-1}}, & p>1,|z|<\frac{\pi z_{0}}{2}, z_{0}=\frac{2 p}{(p-1) \sqrt{a}}, \\
0 \quad & \text { otherwise }
\end{array} .\right.
$$

The solution in (5.4) is known as the compacton solution.

To analyze the stability of (5.4), we linearized the solution of (5.2) near $u_{0}(z)$ and we get

$$
R(U):=-c U^{\prime}+a p\left(u_{0}^{p-1}(z) U\right)^{\prime}+p\left(u_{0}^{p-1}(z) U\right)^{\prime \prime \prime}+\lambda U=0,
$$

where the boundary conditions are $U\left( \pm \frac{p \pi}{(p-1) \sqrt{a}}\right)=0$, and $U^{\prime}$ is periodic. On the other hand the eigenvalue problem is solved here in the unit square in the ac-plane, namely $0<a<1,0<c<1$.

It is worth noticing that when $\lambda=0$, the eigenfunction $U^{(k)}:=u_{0}^{\prime}$, which can be written as

$U=-\frac{\sqrt{a} 2^{\frac{2}{p-1}}\left(\frac{2}{p-1} !\right)}{\left(\frac{4}{p-1} !\right) p}\left(\frac{2 c p}{a(p+1)}\right)^{\frac{1}{p-1}} P_{k}^{k-1}(\xi), \xi=\sin \left(\frac{(p-1) \sqrt{a}}{2 p} z\right), k=\frac{2}{p-1}, p>1$.

It is indeed that after (5.6), the boundary conditions hold when $1<p \leq 2$. We remark that, in this case, the associated Legendre expansion of $u_{0}^{\prime}$ which is given by (5.6) is unique.

However a general eigenfunction assumes one of the following forms;

$$
U^{\left(m_{1}\right)}=-\frac{\sqrt{a} 2^{\frac{2}{p-1}}\left(\frac{2}{p-1} !\right)}{\left(\frac{4}{p-1} !\right) p}\left(\frac{2 c p}{a(p+1)}\right)^{\frac{1}{p-1}} P_{m_{1}}^{m_{1}-1}(\xi), m_{1}=2, \ldots, k, k+1
$$

or

$$
U^{\left(m_{2}\right)}=-\frac{\sqrt{a} 2^{\frac{2}{p-1}}\left(\frac{2}{p-1} !\right)}{\left(\frac{4}{p-1} !\right) p}\left(\frac{2 c p}{a(p+1)}\right)^{\frac{1}{p-1}} P_{k}^{m_{2}-1}(\xi), m_{2}=2, \ldots, k+1
$$

or

$$
U^{\left(m_{3}\right)}=-\frac{\sqrt{a} 2^{\frac{2}{p-1}}\left(\frac{2}{p-1} !\right)}{\left(\frac{4}{p-1} !\right) p}\left(\frac{2 c p}{a(p+1)}\right)^{\frac{1}{p-1}} P_{m_{3}}^{k-1}(\xi), m_{3}=k-1, k+1, \ldots
$$


In a way similar to that done in section 4 , the equation (5.5) is rewritten in the form and $u_{0}(z)$ is given by (5.4). From (5.9) we get an implicit equation for the eigenvalue $\lambda$

$$
\begin{gathered}
-c\left(e^{-\frac{\lambda z}{c}} U\right)^{\prime}+\left(a p\left(u_{0}^{p-1}(z) U\right)^{\prime}+p\left(u_{0}^{p-1}(z) U\right)^{\prime \prime \prime}\right) e^{-\frac{\lambda z}{c}}=0, \\
\lambda=-2 \frac{\int_{-\frac{\pi z_{o}}{2}}^{\frac{\pi z_{o}}{2}}\left((g U)^{\prime \prime \prime}+a(g U)^{\prime}\right) U e^{-\frac{\lambda z}{c}} d z}{\int_{-\frac{\pi z_{o}}{2}}^{\frac{2}{2}} U^{2} e^{-\frac{\lambda z}{c}} d z},
\end{gathered}
$$

where $g=p u_{0}^{p-1}(z)$. We mention that the integrals in the RHS of (5.11) exist.

Indeed the equation (5.11) is a transcendental equation in $\lambda$ which may solve to real, complex or pure imaginary eigenvalues. A direct result from (5.11) is that if $\lambda$ is an eigenvalue then so is $-\lambda$, then $\lambda=0$ is an eigenvalue. This could be seen by rewriting (5.11) in the form

$$
\lambda=2 \frac{\int_{0}^{\frac{\pi z_{o}}{2}}\left((g U)^{\prime \prime \prime}+a(g U)^{\prime}\right) U \sinh \left(\frac{\lambda z}{c}\right) d z}{\int_{0}^{\frac{\pi z_{o}}{2}} U^{2} \cosh \left(\frac{\lambda z}{c}\right) d z} .
$$

It is worth noticing that for the aim of the subsequent computations, the equation ( 5.11) will be rewritten in terms of $\xi$. We mention that when studying the stability of TWS, there may exist more than one stability parameter. This arises from the fact that $\lambda$ depends on the parameters $p, a$, and $c$. So that, we may specify the "dominant parameter", namely a, as to be the stabilization parameter.

First we consider the suggested class of eigenfunctions given by (5.7), they are denoted by $U_{0}^{\left(m_{1}\right)}$. We evaluate the eigenvalues $\lambda_{0}^{\left(m_{1}\right)}$ by using (5.11), we find that $\lambda_{0}^{\left(m_{1}\right)}=0$ for all $m_{1}$. This is a direct consequence of the impaired of the integrand in the upper integral in (5.11). This result means that the eigenvalue $\lambda=0$ is degenerate and it corresponds to infinite eigenfunction ( as it will be seen later ).

As the suggested eigenfunction is not the formal exact ones, we take $m_{1}=1$ and calculate the error norm. To this end we evaluate the relative error-norm $\left\|(R E)_{0}^{(1)}\right\|=\left\|U_{1}^{(1)}-U_{0}^{(1)}\right\|_{2} /\left\|U_{0}^{(1)}\right\|_{2}$, where, for arbitrary $p, a, c$, explicit equations may be calculated directly. But they are cumbersome. As the stabilization parameter was taken a, we fix the values of $p=\frac{3}{2}$, and $c=0.5$. In this case $k=4$ and $m_{1}=2,3,4,5(\mathrm{cf}(5.7))$. Numerical results are carried out for $\left\|(R E)_{0}^{(m)}\right\|$ and the results are shown in Figure 4 when $0<a<1$ and $m_{1}=2,3,4$.

To evaluate $U_{n}^{\left(m_{1}\right)}, n=1,2, \ldots$, we use the variational equation, in which $m=$ $m_{1}$, or $m_{3}$, 


$$
\begin{aligned}
& U_{n+1}^{(m)}=U_{n}^{(m)}+\int_{-1}^{\xi} \eta(y)\left(\frac { ( p - 1 ) ^ { 2 } a } { 4 p } \left(\left(1-y^{2}\right)\left(u_{0}^{p-1}(y) \widetilde{U_{n}^{(m)}(y)}\right)^{\prime \prime \prime}-3 y\left(u_{0}^{p-1}(y) \widetilde{U_{n}^{(m)}(y)}\right)^{\prime \prime}\right.\right. \\
& \left.-\left(u_{0}^{p-1}(y) \widetilde{U_{n}^{(m)}(y)}\right)^{\prime}\right)+a p\left(u_{0}^{p-1}(y) \widetilde{U_{n}^{(m)}(y)}\right)^{\prime} \\
& \left.-c\left(U_{n}^{(m)}(y)\right)^{\prime}+\frac{2 p \lambda_{n}^{(m)}}{(p-1) \sqrt{a} \sqrt{1-y^{2}}} U_{n}^{(m)}(y)\right) d y, n \geq 0 .
\end{aligned}
$$

In (5.13), $\widetilde{U_{n}^{(m)}(y)}$ is taken with a restricted variation; $\delta \widetilde{U_{n}^{(m)}(y)}=0$, so $\eta(y)$ is given by the following differential equation

$$
c \frac{d \eta}{d y}+\lambda \eta=0, \eta(z)=\frac{1}{c} .
$$

By evaluating $U_{1}^{\left(m_{1}\right)}$, and then by substituting into (5.11) to get $\lambda_{1}^{(m)}$, we find that $\lambda_{1}^{\left(m_{1}\right)}=0$, again, due to the impaired in the upper integral. Now, we evaluate $\left\|E_{1}^{\left(m_{1}\right)}\right\|_{R}$, when $m_{1}=2,3,4,5$ and the results are shown in Figure 4.



Figure 4

After Figure 4, we find that $\left\|(R E)_{1}^{\left(m_{1}\right)}\right\|<\left\|(R E)_{0}^{\left(m_{1}\right)}\right\|$ for all $0<a<1$. We mention that when $m_{1}=5=k+1$, then $\left\|(R E)_{n}^{(5)}\right\| \equiv 0$, for all $n$. If, in (5.5) we assume that $m_{1}=k+2, \ldots$, we have also that $\lambda_{0}^{\left(m_{1}\right)}=0$, but computation of $\left\|(R E)_{0}^{\left(m_{1}\right)}\right\|$ shows that it is diverges as $m_{1} \rightarrow \infty$. Thus in the sense of the convergence theorem for the VIM, these cases were discarded.

From the above results, in this case, the eigenvalue $\lambda=0$ is degenerate

Now we consider the equation (5.8) and evaluate the eigenvalues by using the equation (5.11), we find that $\lambda_{0}^{\left(m_{2}\right)}=0, \lambda_{1}^{\left(m_{2}\right)}=0, \ldots, \lambda_{n}^{\left(m_{2}\right)}=0$ for all $n$. When $p=\frac{3}{2}$, the norms of the relative error namely $\left\|(R E)_{0}^{\left(m_{2}\right)}\right\|$, and $\left\|(R E)_{1}^{\left(m_{2}\right)}\right\|$ are given respectively by the following equations when $m_{2}=2,3,4,5$. 


$$
\begin{gathered}
\left\|(R E)_{0}^{\left(m_{2}\right)}\right\|=0.1556 a c^{2}, 0.0337 a c^{2}, 0,0.0159 a c^{2}, \\
\left\|(R E)_{1}^{\left(m_{2}\right)}\right\|=0.1596 a c^{2}, 0.117 a c^{2}, 0,0.005 a c^{2} .
\end{gathered}
$$

When considering the equation (3.9), we find that $\lambda_{n}^{\left(m_{3}\right)}=0$ for all $\mathrm{n}$ and $m_{3}$, but in this case $\left\|(R E)_{n}^{\left(m_{3}\right)}\right\|$ diverges when $n \rightarrow \infty$ or $m \rightarrow \infty$. So that this case is disregarded.

Now, we investigate the existence of pure imaginary eigenvalues $(\lambda= \pm i \omega)$ or real eigenvalues $(\lambda= \pm \mu)$.

To this end and by bearing in mind that $m_{1}$ and $m_{2}$ take the same values, we have interesting results between the norms of the relative error in the cases $\lambda=0, \lambda^{(m)}= \pm i \omega^{(m)}$ and $\lambda= \pm \mu_{0}^{(m)}$. If $\left\|(R E)_{n}^{\left(m_{1}\right)}\right\|,\left\|(R E)_{n}^{\left(m_{2}\right)}\right\|$ and $\left\|(R E)_{n}^{\left(m_{3}\right)}\right\|$ are the relative errors in the three cases respectively. Then the following lemma holds

Lemma 5.2. The following relationships hold: $\left\|(R E)_{0}^{\left(m_{2}\right)}\right\|=\left\|(R E)_{0}^{\left(m_{1}\right)}\right\|+$ $\left.\left(\omega_{0}^{\left(m_{1}\right)}\right)^{2},\left\|(R E)_{0}^{\left(m_{3}\right)}\right\|=\| R E\right)_{0}^{(m)} \|+\left(\mu_{0}^{(m)}\right)^{2}, m=m_{1}=m_{2}$.

The proof is direct as every term is calculated exactly.

As the case when $m=m_{3}$ was ignored, then only the first relation is significant. We find that when $\left(\omega_{0}^{\left(m_{1}\right)}\right)^{2}>1$, then no pure imaginary eigenvalue solutions exist in the sense of the convergence theorem for the VIM. A necessary condition for these eigenvalues to hold is that $\left(\omega_{0}^{\left(m_{1}\right)}\right)^{2}<\left\|(R E)_{1}^{\left(m_{1}\right)}\right\| \mathrm{i} 1 / 2$ and the new errors are still tolerable.

By using the equation (5.15) and from the above arguments, we find that, when $m_{1}=m_{2}=2,3,4,5$

$$
\left(\omega_{0}^{\left(m_{1}\right)}\right)^{2}=0.15754 a c^{2}, 0.1963 a c^{2}, 0,0.2366 a c^{2} .
$$

In case of the equation $(5.16)$, and $\left(m_{2}=2,3,4,5\right)$ we find that; the

$$
\left(\omega_{1}^{\left(m_{1}\right)}\right)^{2}=0.03823 a c^{2}, 0.102 a c^{2}, 0,0 .
$$

By a way similar to that done in the above we could show that complex eigenvalues do not hold.

From these results, only pure imaginary eigenvalues hold for (5.11) (or (5.5)). Thus compacton solutions are stable. 


\section{Conclusions}

The stability of traveling wave solutions for evolution equations of order higher than two had been dealt with by using an extended variational approach which was matched to the VIM. The solution of the eigenvalue problem and stability analysis of Kuramoto-Sivashinsky and K(p,q) equations were considered. Inspecting Some direct results for eigenvalues, against the geometric structure of waves, were done. This hold for fronts or backs. For the other waves-geometric structures, the criteria of divergence of the VIM was used to reject the suggested eigenfunctions and the calculated eigenvalues associated with. In a future work, stability of traveling wave

solutions for vector-evolution equations will be studied.

\section{References}

[1] Murray, J., Mathematical Biology, Second Edition. Springer, Berlin, 1993.

[2] Kapral, R. and Showalter, K. (Eds), Chemical waves and Patterns, Kluwer, Door drecht (1995).

[3] Volpert, A. I. and Volpert, V. A. , Traveling waves solutions of parabolic systems, Transl. Math. Mono. Amer Math. Soc. Providence 140(1994).

[4] Field, R. J. and Burger, M. , Oscillations and Traveling waves in Chemical Systems, J. Wiley, New York (1985).

[5] Evans, J. Nerve axon equations (iii). Stability of the never impulse., Indiana Univ. Math. J. 22(1972), 577-593.

[6] Evans, J. Nerve axon equations (iv). The stable and the unstable impulse., Indiana Univ. Math. J. 24(1975), 1169-1190.

[7] Gardner, R. A. and Jones C. K. R. T. Traveling waves of perturbed diffusion equation arising in a phase field model., India Univ. Math. J. 39(1990), 1197-1222.

[8] Pego, R. and Weinstein, M. Eigenvalues and instability of solitary waves., Phil. Trans. R. Soc. Lond. A, 340(1992), 47-94.

[9] Nii, S. Stability of traveling multiple-front (multiple-back) wave solution of the Fitzhugh-Nagumo equations., SIAM. J. Math. Anal. 28(1997), 1094-1112.

[10] Afendikov, A. L. and Bridges, J. J. Instability of the Hocking-Stewartson pulse and its implications for three-dimensional, Proc. R. Soc. Lond. A. 457(2001), 257-272.

[11] Reddy, S. C. and Trefethen, L. N., Pseudo spectra of the convection diffusion operators., SIAM. Appl. Math. 54(1994), 1634-1649.

[12] Alexander, J. C., Gardner, R. A. and Jones, C. K. R. T. A topological invariant arising in the stability analysis of traveling waves., J. Reine. Angew. Math. 410(1990), 167212.

[13] Nii, S. A topological proof of stability of $N$-front solutions of the Fitzhugh-Nagumo equations., J. Dynam. Diff. Eqns. 11(1999), 515-555.

[14] G. I. Sivashinsky, Acta. 4(1977), 1177. 
[15] Y. Kuramoto and Tsuzuki. Theor. Phys. 55(1976), 356-369.

[16] Y. Kuramoto and T. Yamada, Prog. Theo. Phys. 64 (1978), 346-367.

[17] Luwai Wazzan, A modified Tanh-Coth method for Solving the general Burgers-Fisher and The Kuramoto-Sivashinsky equations. Communications in Non Linear Science and Numerical Simulation, 14(2009), 2646-2652.

[18] John Weiss, M.Tobar and G. Carnevale, The Painleve' for Partial Differential Equations, J. Math. Phy. 24(1983), 552.

[19] Gui-qoing Xu, Zhi-bin Li, PDEP test: a package for the Painleve' test of nonlinear partial differential equations. Applied Mathematics and Computation, 169(2005), 1364-1379.

[20] K. K. Victor, B. B. Thomas and T. C. Kofane, On the exact solution of the SchäferWayne short pulse equation: WKI eigenvalue problem. J. Phys. A, 39(2007), 5585.

[21] H. Sagan; Boundary and eigenvalue problems in mathematical physics, J. Wiley, (1989).

[22] P. Rosenau and J. M. Hyman, Phys. Rev. Lett. 70 (1993), 564.

[23] P. Rosenau, Physica D, 123(1998), 525.

[24] M. Tatari and M. Dehghan, On the convergence of He's variational iteration method, J. comp. Appl. Math., 207 (2007), 121-128.

[25] He, J. H. Variational iteration method. Applied Mathematics and Computation., 114(1999), 699-708.

[26] W.H.Enright, Verifying approximate solutions to differential equations, Comput. Appl. Math.,185(2006), 203-211. 